\title{
ON W-APPROXIMATELY CONTINUOUS PERRON-STIELTJES AND DENJOY-STIELTJES INTEGRAL
}

\author{
D. N. SARKHEL \\ (Received 6 January 1971, revised 29 September 1971) \\ Communicated by E. Strzelecki
}

\section{Introduction}

The aim of the present paper is to introduce a definition of the Perron-Stieltjes integral employing the notion of approximate derivative with respect to a nondecreasing function $\omega$ and to study some of the properties of the integral. Various authors have studied the Perron integral and Perron-Stieltjes integral in different ways, most of which can be found in the references appended in the list of the bibliography. Among them Ridder [10] uses the concept of approximate $\omega$-derivative but he assumes that the monotone function $\alpha$ associated with $\omega$ is continuous. Finally we consider a more general type of integral, the $\omega$-approximately continuous Denjoy-Stieltjes integral, defined descriptively by the method of Saks [11].

Let $\omega$ be a finite non-decreasing function defined on the real line $\Omega$. Following the definitions of Jeffery [6], p. 617, the "outer $\omega$-measure" and the " $\omega$-measure" of a linear set $E$ are here denoted respectively by $\omega^{*}(E)$ and $|E|_{\omega}$.

Throughout the paper we use the following notations. $S$ denotes the set of points of continuity of $\omega, D=\Omega \backslash S, S_{0}$ denotes the union of the pairwise disjoint open intervals on each of which $\omega$ is constant. $S_{0}^{-}$and $S_{0}^{+}$denote respectively the sets of the left and the right end points of the intervals of $S_{0} ; S_{1}=S_{0}^{-} \cup S_{0}^{+}$, $S_{2}=S \cap S_{1}$ and $S_{3}=S \backslash\left(S_{0} \cup S_{2}\right)$. Then the sets $D$ and $S_{1}$ are countable and for any two distinct points $x_{1}, x_{2}$, at least one of which belongs to $S_{3}$, we have $\omega\left(x_{1}\right) \neq \omega\left(x_{2}\right)$.

We require the following known results and definitions.

THEOREM 1.1. ([6], lemma 2, p. 618). Let E be a bounded linear set. Let each point $x$ of $E$ be the left end-point of a sequence of closed intervals $\left[x, x+h_{x, i}\right]$ for which $h_{x, i} \rightarrow 0$ and $\mathscr{I}$ denote the family of all intervals thus associated with the set $E$. Then for every $\varepsilon>0$, there exists a finite family of pairwise disjoint closed intervals $\Delta_{1}, \Delta_{2}, \cdots, \Delta_{N}$ in $\mathscr{I}$ such that 


$$
\sum_{i=1}^{N} \omega^{*}\left(E \cap \Delta_{i}\right)>\omega^{*}(E)-\varepsilon, \Sigma_{i=1}^{N}\left|\Delta_{i}\right|_{\omega}<\omega^{*}(E)+
$$

THEOREM 1.2. This theorem is the analogue of Theorem 1.1. for closed intervals with ' $x$ ' as the right end-point.

Definition 1.1. ([4], \$2, p. 347). Two sets $A$ and $B$ are said to be $\omega$ separated if for every $\varepsilon>0$ there exist open sets $G_{1} \supset A, G_{2} \supset B$ such that $\left|G_{1} \cap G_{2}\right|_{\omega}<\varepsilon$.

TheORem 1.3. ([4], §2; [7], Th. 2.20, p. 59.). Let $A=A_{1} \cup A_{2}$. If $A$ is $\omega$-measurable and $A_{1}, A_{2}$ are $\omega$-separated, then $A_{1}$ and $A_{2}$ are also $\omega$-measurable. Let the bounded sets $A_{1}$ and $A_{2}$ be not $\omega$-separated. If $E_{1} \subset A_{1}, E_{2} \subset A_{2}$ with $\omega^{*}\left(E_{i}\right)$ sufficiently close to $\omega^{*}\left(A_{i}\right), i=1,2$, then $E_{1}$ and $E_{2}$ are not $\omega$ separated.

TheOREM 1.4. ([4], \$2; [7], Th. 3.9, p. 77 (Lusin's Theorem)). Let $f(x)$ be $\omega$-measurable on the $\omega$-measurable set $A$ with $|A|_{\omega}<+\infty$. Then corresponding to every $\varepsilon>0$ there exists a closed set $E \subset A$ with $\left.|A| E\right|_{N}<\varepsilon$ such that $f(x)$ is continuous on $E$ (relative to $E$ ).

\section{2. $\omega$-density and $\omega$-approximate continuity}

Definition 2.1. (cf. [4], §3). Let $A$ be any linear set and $x$ be any given real number. Let $v=[x, x+h], v^{0}=(x, x+h), h>0$. We set

$$
d(x, h)= \begin{cases}\omega^{*}(A \cap v) /|v|_{\omega} & \text { if }|v|_{\omega} \neq 0, \\ 1 & \text { if }|v|_{\omega}=0 \text { and } A \cap v^{0} \neq \varnothing \\ 0 & \text { otherwise. }\end{cases}
$$

Then $\lim _{h \rightarrow 0} \sup d(x, h)$ and $\lim _{h \rightarrow 0} \inf d(x, h)$ are called respectively the upper and lower right $\omega$-densities of $A$ at $x$. If these limits are equal, the common value is the right $\omega$-density of $A$ at $x$. Left $\omega$-densities are defined similarly. If all the four $\omega$-densities of $A$ at $x$ are equal to one another, the common value is the $\omega$-density of $A$ at $x$.

When the $\omega$-density of $A$ at $x$ exists, $x$ is called a point of dispersion $(\omega)$ or a point of density $(\omega)$ according as the $\omega$-density is 0 or 1 .

Note 2.1. If $x \in S_{0}$ and $x$ is a limit point of $A$ on both sides then clearly $x$ is a point of density $(\omega)$ of $A$. If $x \in A \cap D$, then $x$ is a point of density $(\omega)$ of $A$; if $x \in D \backslash A$ then $x$ is a point of dispersion $(\omega)$ of $A$.

In [4] Chakrabarty has defined the $\omega$-density of subsets of $S_{3}$ while our definition is applicable to all linear sets. We can verify that for any two linear sets $A$ and $B$ the theorems 3.1-3.6 of [4] are true. Whenever necessary we shall refer them for the corresponding results of $\omega$-densities according to our definitions. From theorems 3.1, 3.2 and 3.6 [4] we obtain the theorem 
THEOREM 2.1. An arbitrary linear set has $\omega$-density 0 or 1 a.e. $(\omega)$ on $\Omega$.

Definition 2.2. (cf. [1], p. 267, [5], p. 793). Let $\phi(x)$ be a finite function defined on the set $A \subset \Omega$. For any real number $\xi$, the g.l.b. of the real numbers $k$ for which the set

$$
\{x ; x \in A, x \geqq \xi \text { and } \phi(x)>k\}
$$

has $\omega$-density 0 at $\xi$ is denoted by $u_{\omega}^{+}(\phi, \xi)$ or simply by $u_{\omega}^{+}(\xi)$ and is called the upper right approximate limit $(\omega)$ of $\phi(x)$ at $\xi$ relative to $A$. The 1.u.b. of the real numbers $k$ for which the set

$$
\{x ; x \in A, x \geqq \xi \text { and } \phi(x) \leqq k\}
$$

has $\xi$ as a point of dispersion $(\omega)$ is denoted by $l_{\omega}^{+}(\phi, \xi)$ or simply by $l_{\omega}^{+}(\xi)$ and is called the lower right approximate limit $(\omega)$ of $\phi(x)$ at $\xi$ relative to $A$. The upper and lower left approximate limits $(\omega), u_{\omega}^{-}(\xi)$ and $l_{\omega}^{-}(\xi)$, are defined analogously The extended real numbers

$$
u_{\omega}(\xi)=\max \cdot\left\{u_{\omega}^{+}(\xi), u_{\omega}^{-}(\xi)\right\}, l_{\omega}(\xi)=\min \cdot\left\{l_{\omega}^{+}(\xi), l_{\omega}^{-}(\xi)\right\}
$$

are respectively the upper and lower approximate limits $(\omega)$ of $\phi(x)$ at $\xi$ relative to $A$. If $\boldsymbol{u}_{\omega}(\xi)=l_{\omega}(\xi)$, the common value, $\phi_{\omega}(\xi)$, is the $\omega$-approximate limit of $\phi(x)$ at $\xi$. If further $\phi_{\omega}(\xi)=\phi(\xi)$, then $\phi(x)$ is said to be $\omega$-approximately continuous at $\xi$ relative to $A$.

For the remaining part of this section we suppose that $A$ is a fixed subset of $\Omega$ and $\phi(x)$ is a finite function on $A$. To avoid repetition we shall drop the phrase "relative to $A$ ".

THEOREM 2.2. $u_{\omega}(\xi)$ is the g.l.b. of real numbers $k$ for which the set

$$
E^{k}[\phi]=\{x ; x \in A \text { and } \phi(x)>k\}
$$

has $\omega$-density 0 at $\xi ; l_{\omega}(\xi)$ is the l.u.b. of real numbers $k$ for which the set

$$
E_{k}[\phi]=\{x ; x \in A \text { and } \phi(x) \leqq k\}
$$

has $\omega$-density 0 at $\xi$.

Proof. Let $M$ be the g.l.b. of real numbers $k$ for which the set $E^{k}[\phi]$ has $\omega$-density 0 at $\xi$. Then clearly

$$
M \geqq u_{\omega}(\xi) .
$$

If $u_{\omega}(\xi)=\infty$, then equality holds in (1). If $u_{\omega}(\xi)=-\infty$, then $u_{\omega}^{+}(\xi)=u_{\omega}^{-}(\xi)$ $=-\infty$. This implies that for arbitrary large $k>0$ the sets

$$
\{x ; x \in A, x \geqq \xi \text { and } \phi(x)>-k\},\{x ; x \in A, x \leqq \xi \text { and } \phi(x)>-k\}
$$


have $\omega$-density 0 at $\xi$, which gives that $\xi$ is a point of dispersion $(\omega)$ for the set $E^{-k}[\phi]$ so that $M \leqq-k$. Since $k>0$ is arbitrary, $M=-\infty=u_{\omega}(\xi)$.

Now let $-\infty<u_{\omega}(\xi)<+\infty$. Then for any $\varepsilon>0$, the set

$$
\left\{x ; x \in A \text { and } \phi(x)>u_{\omega}(\xi)+\varepsilon\right\}
$$

has $\omega$-density 0 at $\xi$. This gives $M \leqq u_{\omega}(\xi)+\varepsilon$. Since $\varepsilon>0$ is arbitrary, $M \leqq u_{\omega}(\xi)$. So from (1) we have $M=u_{\omega}(\xi)$. This completes the proof of the first part. The proof of the other part is similar.

THEOREM 2.3. $u_{\omega}^{-}(x)=u_{\omega}^{+}(x)$ and $l_{\omega}^{-}(x)=l_{\omega}^{+}(x)$ at almost all points $(\omega)$ of $\Omega$.

The proof is analogous to that of the corresponding result of Chow Shu-Er ([5], p. 795, §2, lemma 1.).

NoTE 2.2. $\phi(x)$ is $\omega$-approximately continuous at each point of the set $A \cap D$.

For, if $\xi \in A \cap D$, then each of the sets

$$
\{x ; x \in A \text { and } \phi(x) \leqq \phi(\xi)-\varepsilon\},\{x ; x \in A \text { and } \phi(x)>\phi(\xi)+\varepsilon\}
$$

has $\xi$ as a point of dispersion $(\omega)$. This implies that

$$
l_{\omega}(\xi) \geqq \phi(\xi)-\varepsilon \text { and } u_{\omega}(\xi) \leqq \phi(\xi)+\varepsilon .
$$

Since $\varepsilon>0$ is arbitrary, we have

$$
u_{\omega}(\xi) \leqq \phi(\xi) \leqq l_{\omega}(\xi) .
$$

Again, $\xi$ is a point of density $(\omega)$ of each of the sets

$$
\{x ; x \in A \text { and } \phi(x)>\phi(\xi)-\varepsilon\},\{x ; x \in A \text { and } \phi(x)<\phi(\xi)+\varepsilon\},
$$

which gives

whence

$$
l_{\omega}(\xi) \leqq \phi(\xi) \leqq u_{\omega}(\xi)
$$

$$
l_{\omega}(\xi)=\phi(\xi)=u_{\omega}(\xi) .
$$

LemMa 2.1. If each point of a set $B$ is a point of density $(\omega)$ of the set $A$, then at any point $\alpha$ the four $\omega$-densities of $A$ are greater than or equal to the corresponding four $\omega$-densities of $B$.

Proof. Let the upper right $\omega$-density of $B$ at $\alpha$ be $k>0$. If $\alpha \in D$, then by Note $2.1 \alpha$ must belong to $B$ and therefore to $A$. If $\alpha \in S_{0} \cup\left(S \cap S_{0}^{-}\right)$, then $\alpha$ must be a limiting point of $B$, and therefore of $A$, on the right. In these cases $A$ and $B$ both have right $\omega$-density unity at $\alpha$.

Next suppose that $\alpha \in S \backslash\left(S_{0} \cup S_{0}^{-}\right)$. Choose $\varepsilon>0$ arbitrarily. Then there exists a strictly decreasing sequence $\left\{\alpha_{n}\right\}$ converging to $\alpha$ such that 


$$
\omega^{*}\left(B \cap\left[\alpha, \alpha_{n}\right]\right) /\left|\left[\alpha, \alpha_{n}\right]\right|_{\omega}>k /(1+\varepsilon), \text { for all } n \text {. }
$$

Let $P_{i}=B \cap\left(\alpha, \alpha_{i}\right]$ and $\varepsilon_{i}=\left|\left[\alpha, \alpha_{i}\right]\right|_{\omega} \cdot k \cdot \varepsilon^{2} /(1+\varepsilon), i=1,2, \cdots$. Take any positive integer $n$. For every $\xi \in P_{n}$ there exists a strictly decreasing sequence of closed intervals $\left[\xi_{i}^{\prime}, \xi\right] \subset(\alpha, \xi]$ with $\xi_{i}^{\prime} \rightarrow \xi$ - such that

$$
\omega^{*}\left(A \cap\left[\xi_{i}^{\prime}, \xi\right]\right) /\left|\left[\xi_{i}^{\prime}, \xi\right]\right|_{\omega}>1-\varepsilon, \quad i=1,2, \cdots .
$$

So by theorem 1.2 there exists a finite number of pairwise disjoint such intervals $\left[\eta_{1}, \xi_{1}\right],\left[\eta_{2}, \xi_{2}\right], \cdots,\left[\eta_{N}, \xi_{N}\right]$ for which

$$
\sum_{i=1}^{N} \omega^{*}\left(P_{n} \cap\left[\eta_{i}, \xi_{i}\right]\right)>\omega^{*}\left(P_{n}\right)-\varepsilon_{n} .
$$

From (2), (3) and (4) we deduce

$$
\begin{aligned}
& \sum_{i=1}^{N} \omega^{*}\left(A \cap\left[\eta_{i}, \xi_{i}\right]\right)>(1-\varepsilon) \cdot \sum_{i=1}^{N}\left|\left[\eta_{i}, \xi_{i}\right]\right|_{\omega} \\
& \quad \geqq(1-\varepsilon) \cdot \Sigma_{i=1}^{N} \omega^{*}\left(P_{n} \cap\left[\eta_{i}, \xi_{i}\right]\right)>(1-\varepsilon) \cdot\left[\omega^{*}\left(P_{n}\right)-\varepsilon_{n}\right] \\
& \quad>(1-\varepsilon) \cdot\left(\varepsilon_{n}-\varepsilon_{n} \cdot \varepsilon^{2}\right) / \varepsilon^{2}=(1-\varepsilon)^{2} \cdot k \cdot\left|\left[\alpha, \alpha_{n}\right]\right|_{\omega} .
\end{aligned}
$$

Since the intervals $\left\{\left[\eta_{i}, \xi_{i}\right]\right\}$ are pairwise disjoint and $\alpha<\eta_{i}<\xi_{i}<\alpha_{n}$, we get from (5),

$$
\omega^{*}\left(A \cap\left[\alpha, \alpha_{n}\right]\right) /\left|\left[\alpha, \alpha_{n}\right]\right|_{\omega}>(1-\varepsilon)^{2} \cdot k, \quad n=1,2, \cdots .
$$

Relation (6) implies that the upper right $\omega$-density of $A$ at $\alpha$ is $\geqq(1-\varepsilon)^{2} \cdot k$. Since $\varepsilon>0$ is arbitrary, it follows that the upper right $\omega$-density of $A$ at $\alpha$ is $\geqq k$.

Similar proofs hold in the other cases.

THEOREM 2.4. The functions $u_{\omega}(x)$ and $l_{\omega}(x)$ are $\omega$-measurable on the real line $\Omega$.

Proof. The functions $u_{\omega}(x)$ and $l_{\omega}(x)$ are defined on $\Omega$ with values in the extended real number system.

Given any real number $a$, let us write

$$
E_{a}=\left\{x ; u_{\omega}(x)<a\right\} \text { and } E^{a}=\left\{x ; u_{\omega}(x) \geqq a\right\} .
$$

We define the sets $A_{1}, A_{2}, \cdots$ by

$$
A_{n}=\left\{x ; u_{\omega}(x)<a-\frac{1}{n}\right\} .
$$

Then $E_{a}=\bigcup_{n=1}^{\infty} A_{n}$. Take a fixed $n$ and write $E=\{x ; x \in A$ and $\phi(x)>a-1 / n\}$. Then by Theorem 2.2 it follows that no point of $E^{a}$ is a point of dispersion $(\omega)$ of $E$. Hence by Theorem $2.1, E$ has $\omega$-density unity at almost all points $(\omega)$ of $E^{a}$, while $E$ has $\omega$-density 0 at each point of $A_{n}$. By Lemma 2.1 it follows easily that $E^{a}$ has $\omega$-density 0 at almost all points $(\omega)$ of $A_{u}$. Therefore $E^{a}$ has $\omega$-density 0 at almost all points $(\omega)$ of $E_{a}$. So by Theorem 3.3. [4] and Theorem 1.3 it follows that the sets $E_{a}$ and $E^{a}$ are $\omega$-measurable. Hence $u_{\omega}(x)$ is $\omega$-measurable on $\Omega$. 
An analogous proof holds for $l_{\omega}(x)$.

Corollary 2.4.1. The functions $u_{\omega}^{-}(x), u_{\omega}^{+}(x), l_{\omega}^{-}(x), l_{\omega}(x)$ are all $\omega-$ measurable on $\Omega$.

PRoOF. By Theorem $2.3 u_{\omega}(x)=u_{\omega}^{+}(x)=u_{\omega}^{-}(x)$ a.e. $(\omega)$ and $l_{\omega}(x)=l_{\omega}^{+}(x)$ $=l_{\omega}^{-}(x)$ a.e. $(\omega)$. These observations in conjunction with Theorem 2.4 establish the corollary.

Corollary 2.4.2. Let $A$ be $\omega$-measurable. Then $\phi(x)$ is $\omega$-measurable on $A$ if, and only if, $\phi(x)$ is $\omega$-approximately continuous a.e. $(\omega)$ on $A$.

Proof. Let $\phi(x)$ be $\omega$-approximately continuous a.e. $(\omega)$ on $A$. Then $\phi(x)=u_{\omega}(x)=l_{\omega}(x)$ a.e. $(\omega)$ on $A$. Since $A$ is $\omega$-measurable, by Theorem 2.4 it follows that $\phi(x)$ is $\omega$-measurable on $A$.

Conversely, let $\phi(x)$ be $\omega$-measurable on $A$. Then using Theorem 1.4 it can be proved in the usual way that $\phi(x)$ is $\omega$-approximately continuous a.e. $(\omega)$ on $A$, remembering that $\phi(x)$ is $\omega$-approximately continuous at each point of $A \cap D$ (Note 2.2.).

THEOREM 2.5. $u_{\omega}^{+}(x) \geqq l_{\omega}^{+}(x)\left[u_{\omega}^{-}(x) \geqq l_{\omega}^{-}(x)\right]$, if the upper right [left] $\omega$-density of $A$ at $x$ is $>0$.

These results follow easily from the definitions.

CoRollary 2.5.1. If $\xi$ be not a point of dispersion $(\omega)$ of $A$, then $l_{\omega}(\xi) \leqq u_{\omega}(\xi)$.

THEOREM 2.6. $l_{\omega}(x) \leqq \phi(x) \leqq u_{\omega}(x)$ a.e. $(\omega)$ on $A$.

Proof. Let $E=\left\{x ; x \in A\right.$ and $\left.\phi(x)>u_{\omega}(x)\right\}$. For any two positive integers $n, k$ let us write

$$
A_{n, k}=\left\{x ; x \in E, \phi(x)-u_{\omega}(x)>\frac{1}{n} \text { and } \phi(x)>-k\right\} .
$$

Suppose, if possible, $\omega^{*}\left(A_{n, k}\right)>0$ for some pair $(n, k)$. Then $A_{n, k}$ contains a component $P$, every point of which is a point of density $(\omega)$ of $P$ and $\omega^{*}(P)=\omega^{*}\left(A_{n, k}\right)$. Now $\phi(x)>-k$ for all $x \in P$, so that $u_{\omega}(x) \geqq-k$. Hence $\phi(x)>(1 / n)-k$ for all $x \in P$. This implies, similarly, $\phi(x)>(2 / n)-k$ for all $x \in P$. Repeating the process we get $\phi(x)>(m / n)-k$ for all $x \in P$ and for every positive integer $m$. This contradicts that $\phi(x)$ is a finite function. Thus $\omega^{*}\left(A_{n k}\right)=0$ for all $(n, k)$, and hence

$$
\omega^{*}(E)=\omega^{*}\left(\bigcup_{n=1}^{\infty} \bigcup_{k=1}^{\infty} A_{n, k}\right)=0 .
$$

Similarly the $\omega$-measure of the set

$$
\left\{x ; x \in A \text { and } \phi(x)<l_{\omega}(x)\right\}
$$

is zero. This proves the theorem. 


\section{Approximate $\omega$-derivatives of functions in the class $\mathscr{F}$}

We denote by $\mathscr{F}$ the class of all finite functions $f(x)$ defined on $\Omega$ such that if $x_{0} \in D$, then $f(x)$ tends to finite limits as $x$ tends to $x_{0}+$ and $x_{0}$ - over the set $S$; these limits are denoted by $f\left(x_{0}+\right)$ and $f\left(x_{0}-\right)$ respectively. If $f \in \mathscr{F}$, we define the function $\bar{f}$ as follows:

$$
\begin{array}{rlrl}
f(x) & =f(x) & & \text { if } x \in S, \\
& =\frac{1}{2}[f(x+)+f(x-)] & \text { if } x \in D .
\end{array}
$$

In this section we consider only the functions of the class $\mathscr{F}$.

Definition 3.1. For any given real number $\xi$ we define the function $\psi(f ; \xi, x)$ by

$$
\psi(f ; \xi, x)= \begin{cases}{[\bar{f}(x)-\bar{f}(\xi)] /[\bar{\omega}(x)-\bar{\omega}(\xi)]} & \text { if } \bar{\omega}(x) \neq \bar{\omega}(\xi), \\ {[f(\xi+)-f(\xi-)] /[\omega(\xi+)-\omega(\xi-)]} & \text { if } x=\xi \in D, \\ 0 & \text { otherwise. }\end{cases}
$$

The upper and lower approximate limits $(\omega)$ of $\psi(f ; \xi, x)$ at $\xi$ are defined to be the upper and lower approximate $\omega$-derivatives of $f$ at $\xi$, and are denoted by $\overline{A D} f_{\omega}(\xi)$ and $A D f_{\omega}(\xi)$ respectively. The right (left) upper and lower approximate $\omega$-derivatives are defined in a corresponding way. They are denoted by $A D^{+} f_{\omega}(\xi), A D_{+} f_{\omega}(\xi), A D-f_{\omega}(\xi)$ and $A D_{-} f_{\omega}(\xi)$ respectively. If $\overline{A D} f_{\omega}(\xi)$ $=A D f_{\omega}(\xi)$, the common value is denoted by $(a p) f_{\omega}^{\prime}(\xi)$ and it is called the approximate $\omega$-derivative of $f(x)$ at $\xi$. If $A D^{+} f_{\omega}(\xi)=A D_{+} f_{\omega}(\xi)$, the common value $(a p) f_{+\omega}^{\prime}(\xi)$ is the right approximate $\omega$-derivative. We define $(a p) f_{-\omega}^{\prime}(\xi)$ analogously.

The ordinary upper and lower right [left] $\omega$-derivatives of $f(x)$ at $\xi$ are respectively the upper and lower limits of $\psi(f ; \xi, x)$ as $x \rightarrow \xi+[x \rightarrow \xi-]$. These are denoted by $D^{+} f_{\omega}(\xi)$ and $D_{+} f_{\omega}(\xi)\left[D^{-} f_{\omega}(\xi)\right.$ and $\left.D_{-} f_{\omega}(\xi)\right]$. If $D^{+} f_{\omega}(\xi)=D_{+} f_{\omega}(\xi)\left[D^{-} f_{\omega}(\xi)=D_{-} f_{\omega}(\xi)\right]$ the common value is denoted by $f_{+\omega}^{\prime}(\xi)\left[f_{-\omega}^{\prime}(\xi)\right]$. If all the four $\omega$-derivatives at $\xi$ are equal to one another, the common value is denoted by $f_{\omega}^{\prime}(\xi)$ and it is called the $\omega$-derivative of $f(x)$ at $\xi$.

It is clear that $f_{\omega}^{\prime}(x)=0$ for every $x \in S_{0}$ and $f_{\omega}^{\prime}(x)=[f(x+)-f(x-)] /$ $[\omega(x+)-\omega(x-)]$ for every $x \in D$.

THEOREM 3.1.

(i) $A D_{-} f_{\omega}(x) \leqq A D^{-} f_{\omega}(x)$

(ii) $A D_{+} f_{\omega}(x) \leqq A D^{+} f_{\omega}(x)$

(iii) $\underline{A D} f_{\omega}(x) \leqq \overline{A D} f_{\omega}(x)$. 
THEOREM 3.2. If $f_{\omega}^{\prime}(\xi)$ exists, then $(a p) f_{\omega}^{\prime}(\xi)$ also exists and $(a p) f_{\omega}^{\prime}(\xi)=f_{\omega}(\xi)$.

THEOREM 3.3.

(i) $A D_{ \pm}(-f)_{\omega}(x)=-A D^{ \pm} f_{\omega}(x)$

(ii) $A D^{ \pm}(c f)_{\omega}(x)=c \cdot A D^{ \pm} f_{\omega}(x) \quad$ if $c>0$

(iii) $A D^{ \pm}(f+g)_{\omega}(x) \leqq A D^{ \pm} f_{\omega}(x)+A D^{ \pm} g_{\omega}(x)$

(iv) $A D_{ \pm}(f+g)_{\omega}(x) \geqq A D_{ \pm} f_{\omega}(x)+A D_{ \pm} g_{\omega}(x)$,

equality holding in (iii) and (iv) if for at least one of the functions $f(x)$ and $g(x)$ the upper and lower approximate $\omega$-derivatives on the appropriate side are equal, it being assumed that the expressions on the right in (iii) and (iv) are meaningful.

The proofs are straightforward and simple.

THEOREM 3.4. If $f(x)$ be $\omega$-measurable on $[a, b]$, then the approximate $\omega$-derivatives of $f(x)$ are also so on $[a, b]$.

Proof. We prove the theorem for $A D^{+} f_{\omega}(x)$. The proofs in other cases are analogous. Since $\left|S_{0} \cup S_{2}\right|_{\omega}=0$ and every subset of $D$ is $\omega$-measurable, $A D^{+} f_{\omega}(x)$ is $\omega$-measurable on $\left(S_{0} \cup S_{2} \cup D\right) \cap[a, b]$. It is, therefore, sufficient to prove that $A D^{+} f_{\omega}(x)$ is $\omega$-measurable on $S_{3}^{\prime}=[a, b] \cap S_{3}$.

Suppose, if possible, that $A D^{+} f_{\omega}(x)$ is not $\omega$-measurable on $S_{3}^{\prime}$. Then from theorem 1.3 it follows that there is a real number $r$ such that the sets

$$
E_{r}=\left\{x ; x \in S_{3}^{\prime} \text { and } A D^{+} f_{\omega}(x)<r\right\}, E^{r}=\left\{x ; x \in S_{3}^{\prime} \text { and } A D^{+} f_{\omega}(x) \geqq r\right\}
$$

are not $\omega$-separated. So by the same theorem there exists $\varepsilon>0$ such that any set $E_{1} \subset E_{r}$ with $\omega^{*}\left(E_{1}\right)>\omega^{*}\left(E_{r}\right)-2 \varepsilon$ is not $\omega$-separated from any set $E_{2} \subset E^{r}$ with $\omega^{*}\left(E_{2}\right)>\omega^{*}\left(E^{r}\right)-2 \varepsilon$. Let

$$
A_{n}=\left\{x ; x \in E_{r} \text { and } A D^{+} f_{\omega}(x)<r-\frac{1}{n}\right\}, \quad n=1,2, \cdots .
$$

Then $A_{n} \subset A_{n+1}$ and $E_{r}=\bigcup_{n=1}^{\infty} A_{n}$. So $\omega^{*}\left(A_{n}\right) \rightarrow \omega^{*}\left(E_{r}\right)$ as $n \rightarrow \infty$. Therefore we can choose a positive integer $N$ such that $\omega^{*}\left(A_{N}\right)>\omega^{*}\left(E_{r}\right)-\varepsilon$. For every $n$ we denote by $B_{n}$ the set of points $x \in E^{r}$ such that for each $x \in B_{n}$ the set

$$
\left\{x^{\prime} ; x^{\prime}>x \text { and }\left[\bar{f}\left(x^{\prime}\right)-\tilde{f}(x)\right] /\left[\bar{\omega}\left(x^{\prime}\right)-\bar{\omega}(x)\right]>r-\frac{1}{2 N}\right\}
$$

has upper right $\omega$-density $>(1 / n)$ at $x$. Then $B_{n} \subset B_{n+1}$ and $E^{r}=\bigcup_{n=l}^{\infty} B_{n}$. So there exists a positive integer $m$ with $\omega^{*}\left(B_{m}\right)>\omega^{*}\left(E^{r}\right)-\varepsilon$. For each $n$, denote by $C_{n}$ the set of points $x \in A_{N}$ for which 


$$
\left[\bar{f}\left(x^{\prime}\right)-\bar{f}(x)\right] /\left[\bar{\omega}\left(x^{\prime}\right)-\bar{\omega}(x)\right]<r-\frac{1}{N},
$$

except possibly for a set $\left\{x^{\prime}\right\}$ with

$$
\frac{\omega^{*}\left(\left\{x^{\prime}\right\} \cap[x, x+h]\right)}{|[x, x+h]|_{\omega}}<\frac{1}{4 m} \text { whenever } 0<h \leqq \frac{1}{n} .
$$

Clearly $C_{n} \subset C_{n+1}$ and $A_{N}=\bigcup_{n=1}^{\infty} C_{n}$. So for some $k$,

$$
\omega^{*}\left(C_{k}\right)>\omega^{*}\left(A_{N}\right)-\varepsilon>\omega^{*}\left(E_{r}\right)-2 \varepsilon .
$$

By the assumption made about the number $\varepsilon$, it now follows that the sets $B_{m}$ and $C_{k}$ are not $\omega$-separated. Hence by theorem 3.6 [4] the set of points of $B_{m}$ at each of which the $\omega$-density of $C_{k}$ is 1 is of outer $\omega$-measure $>0$. Also $f(x)$ is $\omega$-approximately continuous a.e. $(\omega)$ on $[a, b]$. So we can find a point $\xi \in B_{m}$ which is a point of density $(\omega)$ of $C_{k}$ and at which $f(x)$ is $\omega$-approximately continuous. Then from the definition of $B_{m}$ we see that the set

$$
B=\left\{x ; x>\xi \text { and }[\bar{f}(x)-\bar{f}(\xi)] /[\bar{\omega}(x)-\bar{\omega}(\xi)]>r-\frac{1}{2 N}\right\}
$$

has upper right $\omega$-density $>(1 / m)$ at $\xi$. So we can find $h_{0}$ with $0<h_{0}<(1 / k)$ such that

$$
\omega^{*}\left(B \cap\left[\xi, \xi+h_{0}\right]\right)>\frac{1}{m} \cdot\left|\left[\xi, \xi+h_{0}\right]\right|_{\omega} .
$$

Since $\xi \in S_{3}^{\prime}$ we can find $\delta$ with $0<\delta<h_{0}, \xi+\delta \in S$ such that

$$
|[\xi, x]|_{\omega}<\frac{1}{4 m} \cdot\left|\left[\xi, \xi+h_{0}\right]\right|_{\omega}
$$

for any $x$ in $[\xi, \xi+\delta]$. Take an arbitrary but fixed point $x$ of $C_{k} \cap(\xi, \xi+\delta)$ and set

$$
C=\left\{x^{\prime} ;\left[\bar{f}\left(x^{\prime}\right)-\bar{f}(x)\right] /\left[\bar{\omega}\left(x^{\prime}\right)-\bar{\omega}(x)\right] \geqq r-\frac{1}{N}\right\} .
$$

Then by (7) we have

$$
\frac{\omega^{*}(C \cap[x, x+h])}{|[x, x+h]|_{\omega}}<\frac{1}{4 m} \text { when } 0<h \leqq \frac{1}{k} .
$$

Noting that $\xi+h_{0}-x<(1 / k)$ we get, using (9) and (10),

$$
\begin{aligned}
& \omega^{*}\left(C \cap\left[\xi, \xi+h_{0}\right]\right)=\omega^{*}(C \cap[\xi, x])+\omega^{*}\left(C \cap\left[x, \xi+h_{0}\right]\right) \\
& <|[\xi, x]|_{\omega}+\frac{1}{4 m} \cdot\left|\left[x, \xi+h_{0}\right]\right|_{\omega}
\end{aligned}
$$




$$
<\frac{1}{4 m} \cdot\left|\left[\xi, \xi+h_{0}\right]\right|_{\omega}+\frac{1}{4 m} \cdot\left|\left[\xi, \xi+h_{0}\right]\right|_{\omega}=\frac{1}{2 m} \cdot\left|\left[\xi, \xi+h_{0}\right]\right|_{\omega} .
$$

Write $P=B \backslash C$. Then from (8) and (11) we deduce

$$
\frac{1}{2 m} \cdot\left|\left[\xi, \xi+h_{0}\right]\right|_{\omega}<\omega^{*}\left(P \cap\left[\xi, \xi+h_{0}\right]\right) .
$$

Also, using (9), we get

$$
\omega^{*}(P \cap[\xi, \xi+\delta]) \leqq|[\xi, \xi+\delta]|_{\omega}<\frac{1}{4 m} \cdot\left|\left[\xi, \xi+h_{0}\right]\right|_{\omega} .
$$

From (12) and (13) we conclude that there are points $\xi^{\prime} \in P$ with $\xi+\delta<\xi^{\prime}$ $<\xi+h_{0}$. For such a $\xi^{\prime}$ we have simultaneously

$$
\left[\bar{f}\left(\xi^{\prime}\right)-\bar{f}(\xi)\right] /\left[\bar{\omega}\left(\xi^{\prime}\right)-\bar{\omega}(\xi)\right]>r-\frac{1}{2 N}
$$

and

$$
\left[\bar{f}\left(\xi^{\prime}\right)-\bar{f}(x)\right] /\left[\bar{\omega}\left(\xi^{\prime}\right)-\bar{\omega}(x)\right]<r-\frac{1}{N} .
$$

From (14) and (15) we get

$$
\bar{f}\left(x-\bar{f}(\xi)>r \cdot[\bar{\omega}(x)-\bar{\omega}(\xi)]+\frac{1}{2 N} \cdot\left[\bar{\omega}\left(\xi^{\prime}\right)+\bar{\omega}(\xi)-2 \bar{\omega}(x)\right] .\right.
$$

So we have, as $x, \xi \in S$,

$$
f(x)-f(\xi)>r \cdot[\omega(x)-\omega(\xi)]+\frac{1}{2 N} \cdot[\omega(\xi+\delta)+\omega(\xi)-2 \omega(x)] .
$$

The right hand side of (16) tends to $(1 / 2 N) \cdot[\omega(\xi+\delta)-\omega(\xi)]$ as $x \rightarrow \xi+$. So there is a $\delta_{0}\left(0<\delta_{0}<\delta\right)$ such that

$$
f(x)-f(\xi)>\frac{1}{4 N} \cdot[\omega(\xi+\delta)-\omega(\xi)]>0
$$

for all $x \in C_{k} \cap\left(\xi, \xi+\delta_{0}\right)$. Since $C_{k}$ has $\omega$-density 1 at $\xi$, the relation (17) contradicts that $f(x)$ is $\omega$-approximately continuous at $\xi$. This proves the theorem.

THEOREM 3.5. If $f(x)$ be $\omega$-measurable on $[a, b]$, then the $\omega$-derivatives of $f(x)$ are also so on $[a, b]$.

Proof. We prove the theorem for $D^{+} f_{\omega}(x)$. The proofs in other cases are analogous.

As remarked in Theorem 3.4 it is sufficient to show that $D^{+} f_{\omega}(x)$ is $\omega$-measurable on $S_{3}^{\prime}$. Suppose, if possible, that $D^{+} f_{\omega}(x)$ is not $\omega$-measurable on $S_{3}^{\prime}$. Then there is a real number $r$ such that the sets

$$
E_{r}=\left\{x ; x \in S_{3}^{\prime} \text { and } D^{+} f_{\omega}(x)<r\right\}, E^{r}=\left\{x ; x \in S_{3}^{\prime} \text { and } D^{+} f_{\omega}(x) \geqq r\right\}
$$
are not $\omega$-separated. 
Let $\left\{c_{i}\right\}$ be a strictly increasing sequence of real numbers tending to $r$. Let $E_{i k}$ denote the set of points $\xi \in E_{r}$ for which

$$
\frac{\bar{f}(\xi+h)-\bar{f}(\xi)}{\overline{\bar{\omega}}(\xi+h)-\bar{\omega}(\xi)}<c_{i} \text { whenever } 0<h<\frac{1}{k} .
$$

Then for sufficiently large $i$ and $k$ (henceforth fixed) the sets $E_{i k}$ and $E^{r}$ are not $\omega$-separated. This in conjunction with a.e. $(\omega) \omega$-approximate continuity of $f(x)$ and the Theorem 3.6 [4] gives that there is a subset $E \subset E^{r}$ with $\omega^{*}(E)>0$, at each point of which $f(x)$ is $\omega$-approximately continuous and $E_{i k}$ has $\omega$-density 1 . Let $\alpha \in E$ and $c$ be a real number with $c_{i}<c<r$. Since $D^{+} f_{\omega}(\alpha)>c$, there exists $h^{\prime}$ with $0<h^{\prime}<(1 / k)$ such that

$$
\bar{f}\left[\left(\alpha+h^{\prime}\right)-\bar{f}(\alpha)\right] /\left[\bar{\omega}\left(\alpha+h^{\prime}\right)-\bar{\omega}(\alpha)\right]>c .
$$

Since $\alpha \in S$ is a point of density $(\omega)$ of $E_{i k}, \alpha$ is a limit point of $E_{i k}$ on both sides. We take any point $\xi \in\left(\alpha, \alpha+h^{\prime}\right) \cap E_{i k}$ and write $h=\alpha+h^{\prime}-\xi$. Then $0<h<(1 / k)$. The relations (18) and (19) give

and

$$
\bar{f}\left(\alpha+h^{\prime}\right)-\bar{f}(\alpha)>c \cdot\left[\bar{\omega}\left(\alpha+h^{\prime}\right)-\bar{\omega}(\alpha)\right]
$$

$$
\bar{f}(\xi+h)-\bar{f}(\xi)<c_{i} \cdot[\bar{\omega}(\xi+h)-\bar{\omega}(\xi)] .
$$

So we get, as $\xi+h=\alpha+h^{\prime}$ and $\alpha, \xi \in S$,

$$
\begin{aligned}
f(\xi)-f(\alpha) & >\left[\bar{\omega}\left(\alpha+h^{\prime}\right)-\omega(\alpha)\right] \\
\cdot & \left\{c-c_{i} \cdot\left[\bar{\omega}\left(\alpha+h^{\prime}\right)-\omega(\bar{\zeta})\right] /\left[\bar{\omega}\left(\alpha+h^{\prime}\right)-\omega(\alpha)\right]\right\},
\end{aligned}
$$

the right hand side of which tends to $\left[\bar{\omega}\left(\alpha+h^{\prime}\right)-\omega(\alpha)\right] \cdot\left(c-c_{i}\right)>0$, as $\xi \rightarrow \alpha+$ over $E_{i k}$. This contradicts the fact that $f(x)$ is $\omega$-approximately continuous at $\alpha$. This proves the theorem.

THEOREM 3.6. If $f(x)$ is monotone on $[a, b]$, then $f_{\omega}^{\prime}(x)$ exists finitely a.e. $(\omega)$ on $[a, b]$.

This can be proved in the usual way.

THEOREM 3.7. Let $f(x)$ be non-decreasing on each interval of $\left(S_{0} \cup S_{2}\right)$ $\cap[a, b]$. If $A D_{+} f_{\omega}(x) \geqq 0$ on $[a, b), A D_{-} f_{\omega}(x) \geqq 0$ on $(a, b]$, then $\bar{f}(x)$ is nondecreasing on $[a, b]$.

Proof. Let $\alpha, \beta(>\alpha)$ be any two $\mathrm{p}$-ints in $[a, b]$. Choose $\varepsilon>0$ arbitrarily. Let

$$
E=\{x ; x \geqq \alpha \text { and } \psi(f ; \alpha, x) \geqq-\varepsilon\} .
$$

From the hypothesis it is clear that $x \in E$ implies 


$$
\bar{f}(x)-\bar{f}(\alpha) \geqq-\varepsilon \cdot[\bar{\omega}(x)-\bar{\omega}(\alpha)] .
$$

Since $A D_{+} f_{\omega}(\alpha) \geqq 0$, the set $E$ has right $\omega$-density 1 at $\alpha$. So there is a point $\xi_{1}>\alpha$ of $E$ such that

Now let

$$
\omega^{*}\left(E \cap\left[\alpha, \xi_{1}\right]\right) \geqq \frac{1}{2} \cdot\left|\left[\alpha, \xi_{1}\right]\right|_{\omega}
$$

$$
E_{1}=\left\{x ; x \geqq \xi_{1} \text { and } \psi\left(f ; \xi_{1}, x\right) \geqq-\varepsilon\right\} .
$$

Then $x \in E_{1}$ implies

$$
\bar{f}(x)-\bar{f}\left(\xi_{1}\right) \geqq-\varepsilon \cdot\left[\bar{\omega}(x)-\bar{\omega}\left(\xi_{1}\right)\right] .
$$

Taking $x=\xi_{1}$ in (20) and then adding with (21) we get $f(x)-\tilde{f}(\alpha) \geqq-\varepsilon$ - $[\bar{\omega}(x)-\bar{\omega}(\alpha)]$, which shews that $E_{1} \subset E$. Since $A D f_{\omega}\left(\xi_{1}\right) \geqq 0$, the set $E$ has right $\omega$-density 1 at $\xi_{1}$. So there is a point $\xi_{2}>\xi_{1}$ of $E$ such that

$$
\omega^{*}\left(E \cap\left[\xi_{1}, \xi_{2}\right]\right) \geqq \frac{1}{2}\left|\left[\xi_{1}, \xi_{2}\right]\right|_{\omega}
$$

Proceeding thus we obtain a strictly increasing sequence $\left\{\xi_{n}\right\}$ from $E$ such that $\xi_{n}<\beta$ and

$$
\omega^{*}\left(E \cap\left[\xi_{n-1}, \xi_{n}\right]\right) \geqq \frac{1}{2}\left|\left[\xi_{n-1}, \xi_{n}\right]\right|_{\omega}
$$

By (20) we have

$$
\dot{f}\left(\xi_{n}\right)-\bar{f}(\alpha) \geqq-\varepsilon \cdot\left[\bar{\omega}\left(\xi_{n}\right)-\bar{\omega}(\alpha)\right] .
$$

Let $\xi=\lim _{n \rightarrow \infty} \xi_{n}$. If $\xi \in D$, making $n \rightarrow \infty$ in (23) we get

$$
f(\xi-)-\tilde{f}(\alpha) \geqq-\varepsilon \cdot[\omega(\xi-)-\bar{\omega}(\alpha)] .
$$

Also as $f_{\omega}^{\prime}(\xi) \geqq 0$, we have $f(\xi+) \geqq f(\xi-)$. We deduce that $\xi \in E$. Next suppose that $\xi \in S$. Then using (22) we easily deduce that

$$
\omega^{*}\left(E \cap\left[\xi_{n}, \xi\right]\right) \geqq \frac{1}{2} \cdot\left|\left[\xi_{n}, \xi\right]\right|_{\omega}, \quad n=1,2, \cdots
$$

This implies that the upper left $\omega$-density of $E$ at $\xi$ is $\geqq \frac{1}{2}$. If $\xi \notin E$ we must have

$$
\bar{f}(\xi)-\bar{f}(\alpha)<-\varepsilon \cdot[\bar{\omega}(\xi)-\bar{\omega}(\alpha)] .
$$

Hence for all $x \in(\alpha, \xi) \cap E$ we have

$$
\bar{f}(\xi)-\bar{f}(x)<-\varepsilon \cdot[\bar{\omega}(\xi)-\bar{\omega}(x)] .
$$

Since $E$ has upper left $\omega$-density $\geqq \frac{1}{2}$ at $\xi$, the relation (24) contradicts that $A D_{-} f_{\omega}(\xi) \geqq 0$. Hence $\xi \in E$.

If $\xi<\beta$, we cover the interval $[\alpha, \beta]$ by a Lebesgue chain and thus obtain

$$
\tilde{f}(\beta)-\bar{f}(\alpha) \geqq-\varepsilon \cdot[\bar{\omega}(\beta)-\bar{\omega}(\alpha)] .
$$


Since $\varepsilon>0$ is arbitrary the relation (25) gives $\bar{f}(\beta) \geqq \bar{f}(\alpha)$, which proves the theorem.

THEOREM 3.8. Let $E \subset[a, b] \mid S_{0}$ with $|E|_{\omega}=0$ and $k$ be any given positive number. Then there exists a non-decreasing function $\sigma(x)$ on $\Omega$ such that $D_{+} \sigma_{\omega}(x)=+\infty$ for every $x \in E \backslash S_{0}^{-}$and $D_{-} \sigma_{\omega}(x)=+\infty$ for every $x \in E \backslash S_{0}^{+}$ and $\bar{\sigma}(b)=k, \bar{\sigma}(a)=0$.

Proof. Clearly $E \subset S$. Let $\left\{G_{n}\right\}$ be a sequence of open sets containing $E$ with $G_{n+1} \subset G_{n}$ and $\left|G_{n}\right|_{\omega}<\left(1 / 4^{n}\right)$. We define the functions $\mu_{n}(x)$ on $\Omega$ by

and set

$$
\mu_{n}(x)=2^{n} \cdot\left|G_{n} \cap(-\infty, x]\right|_{\omega},
$$

$$
\rho(x)=\sum_{n=1}^{\infty} \mu_{n}(x) .
$$

Then $\rho(x)$ is finite and non-decreasing on $\Omega$. Let $x_{0} \in E \backslash S_{0}^{-}$. For every $n$ there is an open interval $I_{n} \subset G_{n}$ with $x_{0} \in I_{n}$. Let $N(h)$ denote the number of open sets $G_{n}$ which contain the interval $\left[x_{0}, x_{0}+h\right]$. Then it is obvious that $N(h) \rightarrow \infty$ as $h \rightarrow 0+$. So for an arbitrary $h>0$ we have

$$
\begin{aligned}
\rho\left(x_{0}+h\right)-\rho\left(x_{0}\right) & =\sum_{n=1}^{\infty} 2^{n} \cdot\left|G_{n} \cap\left[x_{0}, x_{0}+h\right]\right|_{\omega} \\
& \geqq \sum_{n=1}^{N(h)} 2^{n} \cdot\left|G_{n} \cap\left[x_{0}, x_{0}+h\right]\right|_{\omega} \\
& \geqq 2^{N(h)} \cdot\left|\left[x_{0}, x_{0}+h\right]\right|_{\omega} .
\end{aligned}
$$

Hence it follows that for all $x>x_{0}$,

$$
\bar{\rho}(x)-\bar{\rho}\left(x_{0}\right) \geqq\left[\bar{\omega}(x)-\bar{\omega}\left(x_{0}\right)\right] \cdot 2^{N\left(x-x_{0}\right)},
$$

which implies

$$
D_{+} \rho_{\omega}\left(x_{0}\right)=+\infty \text {. }
$$

Similarly, if $x \in E \backslash S_{0}^{+}$, we can show that $D_{-} \rho_{\omega}(x)=+\infty$. Then the function

$$
\sigma(x)=k \cdot[\rho(x)-\bar{\rho}(a)] /[\bar{\rho}(b)-\bar{\rho}(a)]
$$

fulfills all the conditions of the theorem.

\section{The $\omega$-approximately continuous Perron-Stieltjes Integral}

Definition 4.1. A function $M(x)$ in $\mathscr{F}$ is called a major function of an arbitrary function $f(x)$ on $[a, b]$ if

$$
\bar{M}(a)=0,
$$

(ii) $\underline{A D} M_{\omega}(x)>-\infty$ for all $x \in[a, b]$,

(iii) $A D_{-} M_{\omega}(x) \geqq f(x)$ for every $x \in(a, b] \backslash\left[S_{0} \cup\left(S \cap S_{0}^{+}\right)\right]$ 
and $A D_{+} M_{\omega}(x) \geqq f(x)$ for every $x \in[a, b) \backslash\left[S_{0} \cup\left(S \cap S_{0}^{-}\right)\right]$,

(iv) $M(x)$ is non-decreasing on each interval of $[a, b] \cap\left(S_{0} \cup S_{2}\right)$.

A function $m(x)$ in $\mathscr{F}$ is a minor function of $f(x)$ on $[a, b]$ if

(i') $\quad \bar{m}(a)=0$

(ii') $\overline{A D} m_{\omega}(x)<+\infty$ for all $x \in[a, b]$,

(iii') $A D^{-} m_{\omega}(x) \leqq f(x) \quad$ for all $\quad x \in(a, b] \backslash\left[S_{0} \cup\left(S \cap S_{0}^{+}\right)\right]$

and $A D^{+} m_{\omega}(x) \leqq f(x)$ for all $x \in[a, b) \backslash\left[S_{0} \cup\left(S \cap S_{0}^{-}\right)\right]$,

(iv') $m(x)$ is non-increasing on each interval of $[a, b] \cap\left(S_{0} \cup S_{2}\right)$.

NoTE. The existence of at least one major function and at least one minor function of $f(x)$ on $[a, b]$ guarantees that $f(x)$ is finite on $D \cap[a, b]$.

THEOREM 4.1. If $M(x)$ be a major function and $m(x)$ be a minor function of $f(x)$ on $[a, b]$, then $\bar{M}-\bar{m}$ is non-decreasing on $[a, b]$, and in particular $\bar{M}(b) \geqq \bar{m}(b)$.

Proof. Let $g(x)=M(x)-m(x)$. Then $g \in \mathscr{F}$. By definitions of major and minor functions, $A D M_{\omega}(x)-\overline{A D} m_{\omega}(x)$ is well defined on $[a, b]$. So, using Theorem 3.3 we deduce that $A D_{+} g_{\omega}(x) \geqq 0$ on $[a, b)$ and $A D_{-} g_{\omega}(x) \geqq 0$ on $(a, b]$. Further $g(x)$ is non-decreasing on each interval of $[a, b] \cap\left(S_{0} \cup S_{2}\right)$. Therefore by Theorem $3.7 \bar{g}(x)=\bar{M}(x)-\bar{m}(x)$ is non-decreasing on $[a, b]$. In particular $\bar{M}(b)-\bar{m}(b) \geqq \bar{M}(a)-\bar{m}(a)=0$. This completes the proof of the theorem.

Definition 4.2. Given any function $f(x)$ on $[a, b]$, we define the function $\omega_{f}(x)$ on $\Omega$ as follows:

$$
\begin{aligned}
& \omega_{f}(x)=f(x) \cdot[\omega(x+)-\omega(x-)] \text { if } x \in D \cap[a, b] \text {, } \\
& =0 \quad \text { elsewhere. }
\end{aligned}
$$

Definition 4.3. Let $f(x)$ be any function defined on $[a, b]$ possessing at least one major function $M(x)$ and at least one minor function $m(x)$ on $[a, b]$. Then $f(x)$ is said to be $\omega$-approximately Perron-Stieltjes integrable, in short (APS)-integrable, on $[a, b]$ if $\sup _{m} \bar{m}(b)=\inf _{M} \bar{M}(b)$. The common value is then denoted by $(A P S)-\int_{a}^{b} f d \omega$ and is called the (APS)-integral of $f(x)$ on $[a, b]$.

Since there is no chance of confusion with other types of integrals we delete the prefix $(A P S)$ from our notation of integrals that follow. It is obvious from the deflnition that

THEOREM 4.2. A necessary and sufficient condition that $\int_{a}^{b} f d \omega$ may exist 
is that for every $\varepsilon>0$ there is a major function $M(x)$ and a minor function $m(x)$ of $f(x)$ on $[a, b]$ such that $\bar{M}(b)-\bar{m}(b)<\varepsilon$.

THEOREM 4.3. If $\int_{a}^{b} f d \omega$ exists and if $a<c<b$, then each of the integrals $\int_{a}^{c} f d \omega$ and $\int_{c}^{b} f d \omega$ exists and further

$$
\int_{a}^{c} f d \omega+\int_{c}^{b} f d \omega=\int_{a}^{b} f d \omega
$$

Proof. Let $\varepsilon>0$ be given. Then there exists a major function $M(x)$ and a minor function $m(x)$ of $f(x)$ on $[a, b]$ such that

$$
\bar{M}(b)-\bar{m}(b)<\varepsilon .
$$

Now $M(x)$ and $m(x)$ qualify as major and minor functions of $f(x)$ on $[a, c]$, while $M(x)-\bar{M}(c)$ and $m(x)-\bar{m}(c)$ serve for those on $[c, b]$. Now by Theorem 4.1 and relation (26) we get

$$
\bar{M}(c)-\bar{m}(c) \leqq \bar{M}(b)-\bar{m}(b)<\varepsilon,
$$

and

$$
[\bar{M}(b)-\bar{M}(c)]-[\bar{m}(b)-\bar{m}(c)]<\varepsilon,
$$

which imply the existence of $\int_{a}^{c} f d \omega$ and $\int_{c}^{b} f d \omega$ respectively. Again, by definition we have

and

$$
\bar{m}(c) \leqq \int_{a}^{c} f d \omega \leqq \bar{M}(c)
$$

$$
\bar{m}(b)-\bar{m}(c) \leqq \int_{c}^{b} f d \omega \leqq \bar{M}(b)-\bar{M}(c),
$$

which give on adding

$$
\bar{m}(b) \leqq \int_{a}^{c} f d \omega+\int_{c}^{b} f d \omega \leqq \bar{M}(b)
$$

Also we have

$$
\bar{m}(b) \leqq \int_{a}^{b} f d \omega \leqq \bar{M}(b) .
$$

Relations (26), (27) and (28) give that

$$
\left|\int_{a}^{c} f d \omega+\int_{c}^{b} f d \omega-\int_{a}^{b} f d \omega\right|<\varepsilon
$$

Since $\varepsilon>0$ is arbitrary, it follows that

$$
\int_{a}^{c} f d \omega+\int_{c}^{b} f d \omega=\int_{a}^{b} f d \omega
$$


This completes the proof of the theorem.

Corollary 4.3.1. If $\int_{a}^{b} f d \omega$ exists, then for $a \leqq \alpha<\beta \leqq b, \int_{\alpha}^{\beta} f d \omega$ exists.

THEOREM 4.4. If $\int_{a}^{c} f d \omega$ and $\int_{c}^{b} f d \omega$ both exist, where $a<c<b$, then $\int_{a}^{b}$ fdw exists.

Proof. Let $\varepsilon>0$ be given. Then there is a major function $M_{1}(x)$ and a minor function $m_{1}(x)$ of $f(x)$ on $[a, c]$ such that

$$
\bar{M}_{1}(c)-\bar{m}_{1}(c)<\varepsilon ;
$$

there are major and minor functions $M_{2}(x)$ and $m_{2}(x)$ on $[c, b]$ such that

$$
\bar{M}_{2}(b)-\bar{m}_{2}(b)<\varepsilon \text {. }
$$

Let us define

and

$$
\begin{aligned}
M(x) & =M_{1}(x) \quad \text { for } x \leqq c, \\
& =M_{2}(x)+\bar{M}_{1}(c) \text { for } x>c ;
\end{aligned}
$$

$$
\begin{aligned}
m(x) & =m_{1}(x) \quad \text { for } x \leqq c, \\
& =m_{2}(x)+\bar{m}_{1}(c) \quad \text { for } x>c .
\end{aligned}
$$

Then clearly $M, m \in \mathscr{F}$. We can easily verify that $M(x)$ and $m(x)$ qualify as major and minor functions respectively of $f(x)$ on $[a, b]$. Now we have, using (29) and (30),

$$
\begin{aligned}
\bar{M}(b)-\bar{m}(b) & =\left[\bar{M}_{2}(b)+\bar{M}_{1}(c)\right]-\left[\bar{m}_{2}(b)+\bar{m}_{1}(c)\right] \\
& =\left[\bar{M}_{2}(b)-\bar{m}_{2}(b)\right]+\left[\bar{M}_{1}(c)-\bar{m}_{1}(c)\right]<2 \varepsilon .
\end{aligned}
$$

Hence we conclude that $\int_{a}^{b} f d \omega$ exists.

Definition 4.4. If $\int_{a}^{b} f d \omega$ exists, we define the function $F(x)$ by

$$
F(x)=\left\{\begin{array}{llr}
\int_{a}^{x} f d \omega & \text { if } a<x \leqq b, \\
F(b)+\frac{1}{2} \omega_{f}(b) & \text { if } & x>b, \\
-\frac{1}{2} \omega_{f}(a) & \text { if } & x<a, \\
0 & \text { if } & x=a .
\end{array}\right.
$$

Then $F(x)$ is called the indefinite (APS)-integral of $f(x)$ on $[a, b]$. 
THEOREM 4.5. If $\int_{a}^{b} f d \omega$ exists, then the corresponding indefinite integral $F \in \mathscr{F}$. Specifically, $F(x+)=F(x)+\frac{1}{2} \omega_{f}(x)$ and $F(x-)=F(x)-\frac{1}{2} \omega_{f}(x)$ if $x \in D$.

Proof. It is sufficient to restrict our consideration to $[a, b]$ only. We have $F(a-)=-\frac{1}{2} \omega_{f}(a)=F(a)-\frac{1}{2} \omega_{f}(a)$ and $F(b+)=F(b)+\frac{1}{2} \omega_{f}(b)$. Now choose $\varepsilon>0$ arbitrarily. Then there is a major function $M(x)$ and a minor function $m(x)$ of $f(x)$ on $[a, b]$ such that

$$
\bar{M}(b)-\frac{1}{2} \varepsilon<F(b)<\bar{m}(b)+\frac{1}{2} \varepsilon .
$$

Suppose that $a \in D$. Then by definition 4.1, (iii, iii') we have $m(a+)-m(a-$ ) $\leqq \omega_{f}(a) \leqq M(a+)-M(a-)$. But $\bar{M}(a)=\bar{m}(a)=0$. Hence we deduce,

$$
m(a+) \leqq \frac{1}{2} \omega_{f}(a) \leqq M(a+) .
$$

If $\delta>0$ be sufficiently small and $x \in S \cap(a, a+\delta)$, then we have

$$
m(a+)-\varepsilon<m(x) \leqq M(x)<M(a+)+\varepsilon
$$

and

$$
m(x) \leqq F(x) \leqq M(x) .
$$

From (31), (32), (33) and (34) we deduce that

Hence

$$
\left|F(x)-\frac{1}{2} \omega_{f}(a)\right|<3 \varepsilon \text { for all } x \in S \cap(a, a+\delta) .
$$

$$
F(a+)=\frac{1}{2} \omega_{f}(a)=F(a)+\frac{1}{2} \omega_{f}(a) .
$$

Next, suppose that $b \in D$. Then there is a sufficiently small $h>0$ such that for $x \in S \cap(b-h, b)$,

$$
m(b-)-\frac{1}{2} \varepsilon<m(x) \leqq F(x) \leqq M(x)<M(b-)+\frac{1}{2} \varepsilon .
$$

Also we have

$$
m(b+)-m(b-) \leqq \omega_{f}(b) \leqq M(b+)-M(b-) .
$$

From (31), (35) and (36) we deduce that

$$
-\frac{1}{2} \varepsilon<F(x)-\left[F(b)-\frac{1}{2} \omega_{f}(b)\right]<\frac{1}{2} \varepsilon \text { for all } x \in S \cap(b-h, b) .
$$

Hence $F(b-)=F(b)-\frac{1}{2} \omega_{f}(b)$.

Now let $c \in D \cap(a, b)$. Let $F_{1}(x)$ and $F_{2}(x)$ be respectively the indefinite integrals of $f(x)$ on $[a, c]$ and $[c, b]$. Then $F(x)=F_{1}(x)$ for $x \leqq c$ and $F(x)=F_{2}(x)+F(c)$ for $x \geqq c$. Hence by what has been proved above, we get

$$
F(c-)=F_{1}(c-)=F_{1}(c)-\frac{1}{2} \omega_{f}(c)=F(c)-\frac{1}{2} \omega_{f}(c),
$$

and 


$$
F(c+)=F_{2}(c+)+F(c)=\frac{1}{2} \omega_{f}(c)+F(c) .
$$

This completes the proof of the Theorem.

Corollary 4.5.1. $F_{\omega}^{\prime}(c)=f(c)$ for every $c \in D \cap[a, b]$.

THEOREM 4.6. Let $F(x)$ be the indefinite integral of $f(x)$ on $[a, b]$. Then for any major function $M(x)$ and any minor function $m(x)$ of $f(x)$ on $[a, b]$, the functions $\bar{M}(x)-F(x)$ and $F(x)-\bar{m}(x)$ are non-decreasing on $[a, b]$.

Proof. Let $a \leqq x_{1}<x_{2} \leqq b$. Then $M(x)-\bar{M}\left(x_{1}\right)$ is a major function and $m(x)-\bar{m}\left(x_{1}\right)$ is a minor function of $f(x)$ on $\left[x_{1}, x_{2}\right]$. Now the theorem follows from the inequality

$$
\bar{m}\left(x_{2}\right)-\bar{m}\left(x_{1}\right) \leqq F\left(x_{2}\right)-F\left(x_{1}\right)=\int_{x_{1}}^{x_{2}} f d \omega \leqq \bar{M}\left(x_{2}\right)-\bar{M}\left(x_{1}\right) .
$$

THEOREM 4.7. The (APS)-integral of an arbitrary function $f(x)$ is zero on each closed interval on $S_{0} \cup S_{2}$.

Proof. Let $[a, b]$ be an interval on $S_{0} \cup S_{2}$. Then $M(x) \equiv 0$ qualifies both as a major function and a minor function of $f(x)$ on $[a, b]$. So $\int_{a}^{b} f d \omega=0$.

COROLlary 4.7.1. If $\int_{a}^{a} f d \omega$ exists, then the indefinite integral is constant on each interval of $\left(S_{0} \cup S_{2}\right) \cap[a, b]$.

This follows from Theorems 4.3 and 4.7.

THEOREM 4.8. Let $f \in \mathscr{F}$. If $(a p) f_{\omega}^{\prime}(x)$ exists finitely on $[a, b]$ and $f(x)$ is constant on the intervals of $\left(S_{0} \cup S_{2}\right) \cap[a, b]$, then $\int_{a}^{b}(a p) f_{\omega}^{\prime}(x) d \omega$ exists and has the value $\bar{f}(b)-\bar{f}(a)$.

Proof. The function $f(x)-\bar{f}(a)$ qualifies both as a major function and a minor function of $(a p) f_{\omega}^{\prime}(x)$ on $[a, b]$. Hence the theorem follows.

THEOREM 4.9. Let $\int_{a}^{b} f d \omega$ exist and $F(x)$ be the corresponding indefinite integral. Then $(a p) F_{\omega}^{\prime}(x)$ exists finitely a.e. $(\omega)$ and $(a p) F_{\omega}^{\prime}(x)=f(x)$ a.e. $(\omega)$ on $[a, b]$.

Proof. By Theorem $4.5 F$ belongs to the class $\mathscr{F}$ and by Corollary 4.5 .1 $F_{\omega}^{\prime}(\xi)=f(\xi)$ at every point $\xi \in D \cap[a, b]$. Now let

$$
A=\left\{x ; x \in(a, b) \cap S_{3} \text { and } \underline{A D F_{\omega}}(x)<f(x)\right\} .
$$

Choose $\varepsilon>0$ arbitrarily. For each positive integer $n$, let

$$
A_{n}=\left\{x ; x \in A \text { and } \underline{A D F_{\omega}}(x)<f(x)-\varepsilon / 2^{n}\right\} .
$$

There is a major function $M(x)$ of $f(x)$ on $[a, b]$ such that

$$
\bar{M}(b)-F(b)<\varepsilon^{2} / 2^{2 n+1} \text {. }
$$


Write $R(x)=\bar{M}(x)-F(x)$. Then by Theorem $4.6 R(x)$ is non-decreasing on $[a, b]$ and hence by Theorem $3.6 R_{\omega}^{\prime}(x)$ exists finitely a.e. $(\omega)$ on $[a, b]$. If $x \in A_{n}$, $A D M_{\omega}(x)-A D F_{\omega}(x)=R_{\omega}^{\prime}(x)>\left(\varepsilon / 2^{n}\right)$ provided $R_{\omega}^{\prime}(x)$ exists. Let $E$ denote the set of points of $(a, b) \cap S_{3}$ where $R_{\omega}^{\prime}(x)$ exists finitely. Then

$$
E \cap A_{n} \subset B_{n}=\left\{x ; x \in E \text { and } R_{\omega}^{\prime}(x)>\varepsilon / 2^{n}\right\} .
$$

To each point $\xi \in B_{n}$ we can associate a sequence of closed intervals $\left\{\left[\xi, \xi_{i}\right]\right\}$ with $\xi_{i} \in(a, b) \cap S$ and $\xi_{i} \rightarrow \xi+$ such that

$$
\left[R\left(\xi_{i}\right)-R(\xi)\right] /\left[\omega\left(\xi_{i}\right)-\omega(\xi)\right]>\frac{\varepsilon}{2^{n}}, \quad i=1,2, \cdots .
$$

By Theorem 1.1 we can choose a finite number of disjoint such intervals $\Delta_{1}, \Delta_{2}, \cdots, \Delta_{r}$ for which

$$
\sum_{i=1}^{r} \omega^{*}\left(B_{n} \cap \Delta_{i}\right)>\omega^{*}\left(B_{n}\right)-\varepsilon / 2^{n+1} .
$$

From (37), (38) and (39) we deduce that $\omega^{*}\left(B_{n}\right)<\varepsilon / 2^{n}$, and hence $\omega^{*}\left(A_{n}\right)<\varepsilon / 2^{n}$. But $A=\bigcup_{n=1}^{\infty} A_{n}$. Therefore $\omega^{*}(A)<\varepsilon$. Since $\varepsilon>0$ is arbitrary, it follows that $\omega^{*}(A)=0$.

If $B=\left\{x ; x \in(a, b) \cap S_{3}\right.$ and $\left.\overline{A D} F_{\omega}(x)>f(x)\right\}$, we can show, by introducing a minor function, that $\omega^{*}(B)=0$. Hence if $x \in(a, b) \cap S_{3} \backslash(A \cup B)$ then

$$
\overline{A D} F_{\omega}(x) \leqq f(x) \leqq \underline{A D} F_{\omega}(x),
$$

which gives that $(a p) F_{\omega}^{\prime}(x)$ exists and equals $f(x)$. Since $\left|S_{0} \cup S_{2} \cup A \cup B\right|_{\omega}=0$, we conclude that $(a p) F_{\omega}^{\prime}(x)$ exists and equals $f(x)$ a.e. $(\omega)$ on $[a, b]$.

Now let $R(x)=\bar{M}(x)-F(x)$ and $r(x)=F(x)-\vec{m}(x)$, where $M(x)$ and $m(x)$ are major and minor functions of $f(x)$ on $[a, b]$. Let $C$ denote the set of points in $[a, b]$ at which $(a p) F_{\omega}^{\prime}(x)$ exists and $R_{\omega}^{\prime}(x), r_{\omega}^{\prime}(x)$ both exist finitely. Then $|C|_{\omega}=|[a, b]|_{\omega}$. If $x \in C$, we have

$$
(a p) F_{\omega}^{\prime}(x)=\underline{A D} \bar{M}_{\omega}(x)-R_{\omega}^{\prime}(x)=\underline{A D} M_{\omega}(x)-R_{\omega}^{\prime}(x)>-\infty
$$

and

$$
(a p) F_{\omega}^{\prime}(x)=\overline{A D} \bar{m}_{\omega}(x)+r_{\omega}^{\prime}(x)=\overline{A D} m_{\omega}(x)+r_{\omega}^{\prime}(x)<+\infty .
$$

So $(a p) F_{\omega}^{\prime}(x)$ is finite on $C$. Hence the theorem follows.

THEOREM 4.10. If $\int_{a}^{b} f d \omega$ exists then the corresponding indefinite integral $F(x)$ is $\omega$-approximately continuous on $[a, b]$.

Proof. Let $\varepsilon>0$ be given. Then there is a major function $M(x)$ and a minor function $m(x)$ of $f(x)$ on $[a, b]$ such that for all $x \in[a, b]$,

$$
0 \leqq \bar{M}(x)-F(x)<\varepsilon \text { and } 0 \leqq F(x)-\bar{m}(x)<\varepsilon .
$$


By Note $2.2 F(x)$ is $\omega$-approximately continuous at every point of $D$. Now choose $c \in[a, b] \cap S$ arbitrarily. Since $A D M_{\omega}(c)>-\infty$ and $\overline{A D} m_{\omega}(c)<+\infty$, we can find $K>0$ sufficiently large so that except for a set $\{x\}$ with $\omega$-density 0 at $c$, we have

$$
\begin{array}{rr}
\bar{M}(x)-M(c) \leqq-K \cdot[\bar{\omega}(x)-\omega(c)], & x<c \\
\bar{M}(x)-M(c) \geqq-K \cdot[\bar{\omega}(x)-\omega(c)], & x>c \\
\bar{m}(x)-m(c) \geqq K \cdot[\bar{\omega}(x)-\omega(c)], & x<c \\
\bar{m}(x)-m(c) \leqq K \cdot[\bar{\omega}(x)-\omega(c)], & x>c .
\end{array}
$$

From (40) and (41) we deduce that

$$
|F(x)-F(c)|<\varepsilon+K \cdot|\bar{\omega}(x)-\omega(c)|,
$$

except for a set $\{x\}$ with $\omega$-density 0 at $c$. Since $\bar{\omega}(x) \rightarrow \omega(c)$ as $x \rightarrow c$, it follows that $F(x)$ is $\omega$-approximately continuous at $c$. Hence the theorem follows.

Corollary 4.10.1. Let $f(x)$ be (APS)-integrable on $[a, b]$. Then $f(x)$ is necessarily $\omega$-measurable and finite a.e. $(\omega)$ on $[a, b]$.

This follows from Theorems 4.9, 4.10, 3.4 and Corollary 2.4.2.

COROLLARY 4.10.2. If $f(x)$ be (APS)-integrable on $[a, b]$ then the major and minor functions of $f(x)$ possess finite approximate $\omega$-derivatives a.e. $(\omega)$ on $[a, b]$.

THEOREM 4.11. If $\int_{a}^{b} f d \omega$ exists and $g(x)=f(x)$ a.e. $(\omega)$ on $[a, b]$, then $\int_{a}^{b} g d \omega$ exists and $\int_{a}^{b} g d \omega=\int_{a}^{b} f d \omega$.

Proof. Let $E$ denote the set of points of $[a, b] \backslash S_{0}$ for which $\left.g(x) \neq f x\right)$. Then $|E|_{\omega}=0$. So given $\varepsilon>0$ there exists, by Theorem 3.8, a non-decreasing function $\sigma(x)$ with $\bar{\sigma}(b)=\varepsilon$ and $\bar{\sigma}(a)=0$. There are major and minor functions of $f(x)$ on $[a, b]$ such that $\bar{M}(b)-\bar{m}(b)<\varepsilon$. Then $M(x)+\sigma(x)$ and $m(x)-\sigma(x)$ are major and minor functions of $g(x)$ on $[a, b]$ such that $[\bar{M}(b)+\bar{\sigma}(b)]$ $-[\bar{m}(b)-\bar{\sigma}(b)]<3 \varepsilon$. Since $\varepsilon>0$ is arbitrary, it follows that $\int_{a}^{b} g d \omega$ exists. Finally, the relation $\bar{m}(b)-\varepsilon=\bar{m}(b)-\bar{\sigma}(b) \leqq \int_{a}^{b} g d \omega \leqq \bar{M}(b)+\bar{\sigma}(b)=\bar{M}(b)+\varepsilon$ gives that $\int_{a}^{b} g d \omega=\int_{a}^{b} f d \omega$.

THEOREM 4.12. Let $f(x)$ be Lebesgue-Stieltjes summable with respect to $\omega(x)$ on $[a, b]$. Then $(A P S)-\int_{a}^{b} f d \omega$ exists and

$$
(A P S)-\int_{a}^{b} f d \omega=(L S) \int_{a}^{b} f d \omega-\frac{1}{2}\left[\omega_{f}(a)+\omega_{f}(b)\right] .
$$

THEOREM 4.13. If $f(x)$ be non-negative and (APS)-integrable on $[a, b]$ then it is $(L S)$-summable on $[a, b]$. 
Proofs of these two theorems are analogous to those of the corresponding theorems 1 and 2 in [9] (p. 168-169).

\section{5. $A C-\omega$ and $A C G-\omega$ functions belonging to the class $\mathscr{F}$}

Definition 5.1. A function $F \in \mathscr{F}$ is said to be $A C-\omega$ below, $[A C-\omega$ above] on a set $E$ if for every $\varepsilon>0$ there exists $\delta>0$ such that for every finite set of non-overlapping intervals $\left\{\left(x_{i}, x_{i}^{\prime}\right)\right\}$ with end-points on $E$ and with $\Sigma\left|\left[x_{i}, x_{i}^{\prime}\right]\right|_{\omega}<\delta$, we have

$$
\sum\left\{F\left(x_{i}^{\prime}\right)-F\left(x_{i}\right)\right\}>-\varepsilon,\left[\sum\left\{F\left(x_{i}^{\prime}\right)-F\left(x_{i}\right)\right\}<\varepsilon\right] .
$$

$F(x)$ is said to be $A C G-\omega$ below [ $A C G-\omega$ above] on $E$ if $E$ is the union of a countable number of sets on each of which $F(x)$ is $A C-\omega$ below $[A C-\omega$ above]. $F(x)$ is $A C G-\omega$ on $E$ if it icboth $A C G-\omega$ below and $A C G-\omega$ above on $E$.

THEOREM 5.1. (cf. [8], lemma 4.1, p. 261). If $A D F_{\omega}(x)>-\infty$ on $E$ and $F(x)$ be non-decreasing on $E \cap I$ for every interval $\overline{I \text { on }}$ which $\omega(x)$ is constant, then $F(x)$ is $A C G$ - $\omega$ below on $E$.

THEOREM 5.2. (cf. [8], p. 263, lines 2, sqq.). The indefinite integral of an (APS)-integrable function on $[a, b]$ is $A C G-\omega$ on $[a, b]$.

THEOREM 5.3. (cf. [8], lemma 2.1, p. 254). A function $F(x)$ which is $A C G-\omega$ on $[a, b]$ necessarily fulfills the condition $(N \omega)$, that is $|F[H]|=0$ for every set $H \subset[a, b]$ with $|H|_{\omega}=0$.

The definitions adopted in this article are analoguos to those in Yôto Kubota's paper [8]. The proofs of the Theorems 5.1-5.3 are also analogous to those of the corresponding results in [8]. Kubota, with his notion of $A C G$, has defined the $A D$-integral $([8], \S 2)$ by the method of Saks [11]. We show that the same method leads us to the " $\omega$-approximately continuous Denjoy-Stieltjes Integral" or "the (ADS)-integral",

THEOREM 5.4. Let $F \in \mathscr{F}$ and be such that

(i) $F(x)=F(x)$ and $F(x)$ is $\omega$-approximately continuous on $[a, b]$,

(ii) $F(x-) \leqq F(x+)$ for all $x \in D \cap[a, b]$,

(iii) $F(x)$ is non-decreasing on the intervals of $\left(S_{0} \cup S_{2}\right) \cap[a, b]$,

(iv) $F[E]$ contains no interval, where

$$
E=\left\{x ; x \in[a, b] \backslash\left(S_{0} \cup S_{2}\right) \text { and } A D_{-} F_{\omega}(x) \leqq 0\right\}
$$

Then $F(x)$ is non-decreasing on $[a, b]$. 
Proof. Suppose, if possible, that $a \leqq c_{0}<d_{0} \leqq b$, while $F\left(d_{0}\right)<F\left(c_{0}\right)$. As $F[E]$ contains no interval there is $y_{0}$ such that $F\left(d_{0}\right)<y_{0}<F\left(c_{0}\right), y_{0} \notin F[E]$. Let $A=\left\{x ; x \in\left[c_{0}, d_{0}\right]\right.$ and $\left.F(x)>y_{0}\right\}$. Then $c_{0} \in A$. As $F(x)$ is non-decreasing on the intervals of $\left(S_{0} \cup S_{2}\right) \cap[a, b]$ and $F(x)$ is $\omega$-approximately continuous at every point of $[a, b]$, so $A$ has right $\omega$-density 1 at every point of $A$. We can therefore find $c_{n} \in A \cap S$ such that

$$
\omega^{*}\left(A \cap\left[c_{n-1}, c_{n}\right]\right) \geqq \frac{1}{2}\left|\left[c_{n-1}, c_{n}\right]\right|_{\omega},
$$

$c_{0}<c_{1}<c_{2}<\cdots<d_{0}$. Let $c_{n} \rightarrow \alpha$. If $\alpha \in D$, then as $F\left(c_{n}\right)>y_{0}$ for all $n$, so $F(\alpha-) \geqq y_{0} \cdot \operatorname{So} F(\alpha) \geqq y_{0}$. If $F(\alpha)=y_{0}$, then clearly $F(\alpha-)=F(\alpha+)=y_{0}$. This means $F_{\omega}^{\prime}(\alpha)=0$, which contradicts that $y_{0} \notin F[E]$. Thus $F(\alpha)>y_{0}$. If $\alpha \in S$, then we have

$$
\omega^{*}\left(A \cap\left[c_{n}, \alpha\right]\right) \geqq \frac{1}{2} \cdot \sum_{r=n}^{\infty}\left|\left[c_{r}, c_{r+1}\right]\right|_{\omega} \geqq \frac{1}{2} \cdot\left|\left[c_{n}, \alpha\right]\right|_{\omega}
$$

for all $n=1,2,3, \cdots$. So the upper left $\omega$-density of $A$ at $\alpha$ is $>0$. We assert again that $F(\alpha)>y_{0}$. Suppose that $F(\alpha)=y_{0}$. Then for all $x \in A \cap\left[c_{0}, \alpha\right]$ we have

$$
[F(\alpha)-F(x)] /[\bar{\omega}(\alpha)-\bar{\omega}(x)]<0,
$$

so that $A D_{-} F_{\omega}(\alpha) \leqq 0$, which contradicts that $y_{0} \notin F[E]$. If $F(\alpha)<y_{0}$, then as $F(x)$ is $\omega$-approximately continuous at $\alpha$, the set $A$ should have $\omega$-density 0 at $\alpha$, which is not the case. Thus in all cases $\alpha \in A$. If $\alpha<d_{0}$, covering $\left[c_{0}, d_{0}\right]$ by a Lebesgue chain of intervals we get $F\left(d_{0}\right)>y_{0}$, leading to a contradiction. This final contradiction establishes the theorem.

This theorem enables us to enunciate the following.

Definition 5.2. Let $f(x)$ be any function defined on $[a, b]$ and suppose that there exists a function $F \in \mathscr{F}$ such that

(i) $F(x)$ is $\omega$-approximately continuous on $[a, b], F(x)=F(x)$ on $D \cap[a, b]$,

(ii) $F(x)$ is $A C G-\omega$ on $[a, b]$ and $F(x)$ is constant on the intervals of $\left(S_{0} \cup S_{2}\right) \cap[a, b]$,

and

(iii) (ap) $F_{\omega}^{\prime}(x)$ exists finitely a.e. $(\omega)$ on $[a, b]$ and $(a p) F_{\omega}^{\prime}(x)=f(x)$ a.e. $(\omega)$ on $[a, b]$.

Then $f(x)$ is said to be integrable in the w-approximately continuous DenjoyStieltjes sense or (ADS)-integrable and we write

$$
(A D S)-\int_{a}^{b} f d \omega=F(b)-F(a) .
$$

The function $F(x)$ is said to be an indefinite (ADS)-integral of $f(x)$ on $[a, b]$. 
Definition 5.2 requires a uniqueness theorem which is furnished below.

THEOREM 5.5. If $F_{1}(x)$ and $F_{2}(x)$ are any two indefinite (ADS)-integrals of $f(x)$ on $[a, b]$, then $F_{2}(b)-F_{2}(a)=F_{1}(b)-F_{1}(a)$.

Proof. Let $\varepsilon>0$ be given. Consider the function $F(x)=F_{1}(x)-F_{2}(x)$ $+\varepsilon \cdot \bar{\omega}(x)$. Then $F(x) \equiv F(x)$ is $\omega$-approximately continuous on $[a, b]$ and $(a p) F_{\omega}^{\prime}(x)=\varepsilon>0$ a.e. $(\omega)$ on $[a, b]$. So the set

$$
E=\left\{x ; x \in[a, b] \text { and } A D_{-} F_{\omega}(x) \leqq 0\right\}
$$

is of $\omega$-measure zero. But $F(x)$ is $A C G-\omega$ on $[a, b]$. So by Theorem 5.3 $|F[E]|=0$. Hence $F[E]$ contains no interval. At the points of $D \cap[a, b]$, we have $F_{\omega}^{\prime}(x)=\varepsilon>0$. So $F(x+)>F(x-)$. Also $F(x)$ is non-decreasing on the intervals of $\left(S_{0} \cup S_{2}\right) \cap[a, b]$. Hence by Theorem 5.4 we have

$$
F_{1}(b)-F_{1}(a) \geqq F_{2}(b)-F_{2}(a)-\varepsilon \cdot[\bar{\omega}(b)-\bar{\omega}(a)] .
$$

Since $\varepsilon>0$ is arbitrary, it follows that

$$
F_{1}(b)-F_{1}(a) \geqq F_{2}(b)-F_{2}(a) .
$$

Interchanging $F_{1}$ and $F_{2}$ we get,

$$
F_{2}(b)-F_{2}(a) \geqq F_{1}(b)-F_{1}(a) .
$$

Combining (42) and (43) we get the required result.

We note that an (APS)-integrable function is necessarily (ADS)-intergrable and the two integrals have equal value. It is easy to verify that the ADS-integral possesses all the usual fundamental properties of an integral.

In conclusion I express my gratitude to Dr. P. C. Bhakta for his kind suggestions in the preparation of this paper.

\section{References}

[1] H. Blumberg, 'The measurable boundaries of an arbitrary function', Acta Math. 65 (1935), 263-282.

[2] J. C. Burkill, 'The approximately continuous Perron Integral', Math. Zeit. 34 (1931) 270-278.

[3] J. C. Burkill and U.S. Haslam Jones, 'The derivates and approximate derivates of measurable functions', Proc. Lond. Math. Soc. (2) 32 (1931), 346-355.

[4] M. C. Chakrabarty, 'Some results on $\omega$-derivatives and $B V-\omega$ functions', J. Aust. Math. Soc. 9 (1969), 345-360.

[5] Shu-ER Chow, 'On approximate derivatives', Bull. Amer. Math. Soc. 54 (1948), 793-802.

[6] R. L. Jeffery, 'Generalized integrals with respect to functions of bounded variation', Canad. J. Math. 10 (1958), 617-626.

[7] R. L. Jeffery, The theory of functions of a real variable (University of Toronto Press, 1962). 
[8] Yôto Kubota, 'On the approximately continuous Denjoy Integrals', Tohoku Math.Jour. 15 (1963), 253-264.

[9] I. P. Natanson Theory of functions of a real variable, Vol. II. (New York, 1960), Ungar.

[10] J. Ridder, 'Ueber Perron - Stieltjessche und Denjoy-Stieltjessche Integrationen', Math. Zeit. 40 (1936), 127-160.

[11] S. Saks, Theory of the integral, (Dover, New York, 1964).

[12] G. Sonouchi and M. Utagawa, 'The generalized Perron Integrals', Tohoku Math. Jour. 1 (1949), 95-99.

Department of Mathematics

R. K. Mission Vidyamandira

Belur Math. Howrah

West-Bengal, India. 